\title{
Hemangioma infantil: actualización del tratamiento tópico y sistémico
}

\author{
Orly Cheirif-Wolosky ${ }^{1}$, Alma D. Novelo-Soto ${ }^{2}$, Luz Orozco-Covarrubias ${ }^{1}$ y Marimar Sáez-de-Ocariz ${ }^{1 *}$

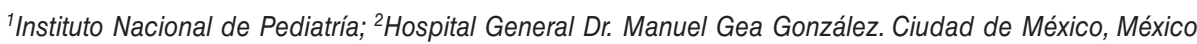

\section{Resumen}

Los hemagiomas infantiles $(\mathrm{HI})$ son los tumores de tejidos blandos más frecuentes de la infancia. Se caracterizan por un crecimiento significativo durante los primeros meses de vida, seguido de una involución lenta y espontánea a lo largo de un periodo que puede durar algunos años. Usualmente, la regresión de la mayor parte del tumor termina a los 4 años de edad. Sin embargo, algunos de los HI desarrollan complicaciones, lo que resulta en alteraciones funcionales, dolor y desfiguramiento. La decisión de administrar tratamiento a un paciente con HI y elegir la mejor opción terapéutica para ese paciente (tratamiento tópico o sistémico) debe ser individualizada, dependiendo de varios factores: el tamaño de la lesión, la localización, la presencia de complicaciones como ulceración, el riesgo de cicatrización o desfiguramiento, la edad del paciente, la tasa de crecimiento o de involución al momento del diagnóstico, los riesgos y beneficios de administrar el tratamiento, la disponibilidad del medicamento, los costos y la experiencia del médico tratante.

Palabras clave: Hemangioma infantil. Propranolol. Timolol.

\section{Infantile hemangioma: an update in the topical and systemic treatments}

\begin{abstract}
Infantile hemagiomas $(\mathrm{IH})$ are the most common soft tissue tumors in infancy. They are characterized by significant growth during the first months of life, followed by slow spontaneous involution over the ensuring years. The process of involution takes several years, but usually the regression of most of the tumors ends at 4 years of age. Unfortunately, some of the IH develop complications, resulting in functional impairment, pain and disfigurement. The decision to start treatment and the choice of the best therapeutic option (topic or systemic) should be individualized depending on several factors: the size of the lesion, the location, the presence of complications such as ulceration, the risk of scarring or disfigurement, the age of the patient, the rate of growth or regression at the time of diagnosis, the risks and benefits of the treatment, the availability of the medication, the costs, and the experience of the attending physician.
\end{abstract}

Key words: Infantile hemangioma. Propranolol. Timolol.

Disponible en internet: 11-06-2019 Bol Med Hosp Infant Mex. 2019;76:167-175 www.bmhim.com 


\section{Introducción}

Los hemagiomas infantiles $(\mathrm{HI})$ son los tumores de tejidos blandos más frecuentes de la infancia. Están presentes del 4 al $5 \%$ de la población pediátrica ${ }^{1}$ y predominan en el sexo femenino, en una proporción de 1.4:1 a $3: 1^{2,3}$. Estas neoformaciones vasculares se caracterizan por la proliferación anormal de células endoteliales y una arquitectura aberrante de los vasos sanguíneos ${ }^{4}$.Dentro de los factores de riesgo para su desarrollo se encuentran el ser producto de una madre añosa, de gestación múltiple o de parto pretérmino, el sexo femenino, el color de piel (blanco), el bajo peso al nacer y la presencia de anomalías placentarias $^{5}$.

Los $\mathrm{HI}$ generalmente aparecen antes de las 4 semanas de vida y terminan la mayor parte de su crecimiento hacia los 5 meses de edad, aunque esta fase puede extenderse hasta el año de edad 6 . Los HI presentan dos fases de dinámicas de evolución: la proliferación y la involución. La proliferación se presenta durante la infancia temprana y la involución gradual y espontánea inicia cerca del año de edad ${ }^{7}$. Existe un periodo intermedio entre la proliferación y la involución durante la infancia media a tardía que se conoce como meseta, y podría corresponder a un periodo de equilibro entre las células que están proliferando y las que están involucionando. El proceso de involución toma varios años y varía en duración, aunque la regresión de la mayor parte del tumor usualmente termina a los 4 años de edad ${ }^{6}$.

Debido a la historia natural de involución espontánea, la mayoría de los $\mathrm{HI}$ no requieren tratamiento. Sin embargo, algunos pueden comprometer la visión, la audición o la vía aérea, o causar complicaciones como ulceraciones o desfiguración facial ${ }^{8}$. El paciente recibe tratamiento para $\mathrm{HI}$ cuando es electivo para reducir la posibilidad de desfiguración permanente 0 ante alguna de las siguientes condiciones: presencia de complicaciones que ponen en peligro la vida, existencia de alteraciones funcionales, dolor o sangrado ${ }^{6}$.

El tratamiento para los $\mathrm{HI}$ puede ser tópico o sistémico. El tópico suele utilizarse para hemangiomas pequeños, superficiales, o para aquellos pacientes en los cuales el tratamiento sistémico está contraindicado. Por otro lado, el tratamiento sistémico está indicado en hemangiomas grandes, con riesgo de alteraciones funcionales o desfiguramiento y aquellos refractarios a otros tratamientos ${ }^{6,8}$.

\section{Tratamiento tópico}

A continuación, se describen algunos de los tratamientos tópicos que se han utilizado para el manejo del HI (Tabla 1).

\section{Timolol}

Es un $\beta$-bloqueador no cardioselectivo, utilizado por primera vez con éxito en 2010 en el tratamiento de un hemangioma localizado en el párpado superior de una paciente de 4 meses de edad ${ }^{9}$. A partir de entonces, se han publicado diversos reportes de caso y estudios prospectivos con respuestas satisfactorias entre las 4 y 5 semanas posteriores al inicio del tratamiento, con disminución significativa del volumen de la lesión y de su crecimiento y mejoría de la apariencia clínica ${ }^{9-11}$.

La dosis recomendada de maleato de timolol al $0.5 \%$ en solución es una gota (con $0.25 \mathrm{mg}$ ) cada $12 \mathrm{~h}$ hasta por 24 semanas. Recientemente, Fernández-Ballesteros, et al. ${ }^{12}$ publicaron un reporte de caso donde se utilizó una menor concentración del fármaco, con diferente vehículo y con una respuesta excelente. En su reporte se describe el manejo de un hemangioma en el párpado superior, de características similares a las reportadas previamente en la literatura, utilizando timolol gel al $0.1 \%$ oclusivo dos veces al día. El efecto clínico fue apreciable después de los primeros 7 días de tratamiento y la desaparición completa de la lesión se produjo en 4 meses, sin evidencia de efectos adversos locales o sistémicos.

El timolol tópico se recomienda para el tratamiento de hemangiomas superficiales, localizados, no ulcerados, sin afección a mucosas. Chan, et al. ${ }^{13}$, a partir de un estudio clínico aleatorizado donde incluyeron 41 pacientes, observaron que el tratamiento con timolol es más efectivo cuando la lesión tiene un diámetro $<11.3 \mathrm{~mm}$. A la fecha, no se han reportado efectos adversos por la administración tópica (Fig. 1).

\section{Propranolol}

Es un antagonista no cardioselectivo del receptor $\beta$-adrenérgico. Se ha utilizado con éxito tras la administración del ungüento al $0.1 \%$ cada $12 \mathrm{~h}$ durante 10 meses, en especial como terapia adyuvante al tratamiento con láser de colorante pulsado ${ }^{14}$. En las series de casos reportados, el propranolol tópico ha sido efectivo en el manejo de hemangiomas superficiales, y ha inducido la regresión de la lesión y el cese del crecimiento. Los efectos clínicos se observan a los 
Tabla 1. Principales tratamientos tópicos para los hemangiomas infantiles ${ }^{12,14,19-20,23,24,26}$

\begin{tabular}{|c|c|c|}
\hline Fármaco & Dosis & Efectos adversos \\
\hline Timolol al 0.5\% (solución) & $0.25 \mathrm{mg}$ (una gota) cada $12 \mathrm{~h}$ & Sin efectos adversos reportados \\
\hline Propranolol al $0.1 \%$ (ungüento) & Aplicar cada $12 \mathrm{~h}$ & Sin efectos adversos reportados \\
\hline Imiquimod al 5\% (crema) & $\begin{array}{l}\text { No estandarizado } \\
\text { Tres a siete veces por semana }\end{array}$ & Irritación local, hiperpigmentación, costras \\
\hline $\begin{array}{l}\text { Dipropionato de betametasona al } 0.05 \% \text { (crema) } \\
\text { Propionato de clobetasol al } 0.05 \% \text { (crema) } \\
\text { Propionato de halobetasol al } 0.05 \% \text { (crema) } \\
\text { Furoato de mometasona al } 0.1 \% \text { (crema) }\end{array}$ & Aplicar cada $12 \mathrm{~h}$ & Atrofia, ulceración, telangiectasias \\
\hline Brimonidina al $0.2 \% /$ timolol al $0.5 \%$ (solución) & Una a dos gotas cada $12 \mathrm{~h}$ & $\begin{array}{l}\text { Mareo, letargia, fatiga, hipotermia, bradicardia } \\
\text { e hipotensión } \\
\text { Efectos severos: depresión respiratoria y } \\
\text { neurológica }\end{array}$ \\
\hline
\end{tabular}

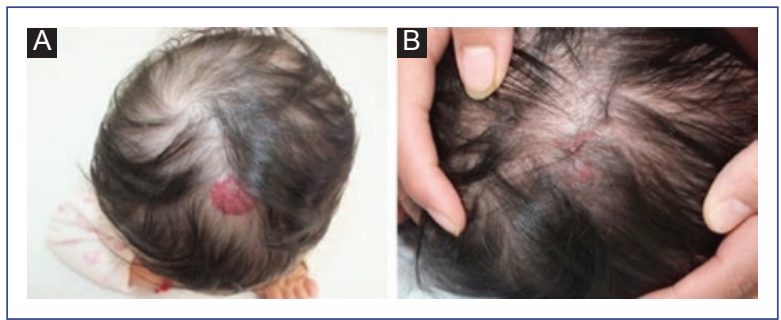

Figura 1. Hemangioma infantil superficial en piel cabelluda. A: previo a tratamiento con timolol. B: después de tres meses de tratamiento con timolol.

2 a 3 meses posteriores al inicio del tratamiento. Cuando se utiliza en combinación con el láser de luz pulsada se ha encontrado beneficio clínico en un tiempo menor, entre 3 y 6 semanas posteriores a su uso. No se han reportado efectos adversos debido a su escasa absorción sistémica, incluso en pacientes prematuros incluidos en los diferentes estudios ${ }^{15-17}$.

\section{Imiquimod}

Es un modificador de la respuesta inmunitaria que actúa sobre el sistema inmunológico innato y adaptativo. Posee propiedades antiangiogénicas y proapoptóticas, estimulando la producción de citocinas proinflamatorias, como el interferón alfa (IFN- $\alpha$ ), interleucina 6 (IL-6) y factor de necrosis tumoral alfa, así como incrementando la actividad de las células natural killer y su efecto en la apoptosis celular ${ }^{18}$.

Existen muy pocos estudios sobre la eficacia del imiquimod en el manejo de hemangiomas. Qiu, et al. ${ }^{19}$ realizaron un estudio retrospectivo donde compararon la eficacia del tratamiento tópico con imiquimod al 5\% vs. timolol al $0.5 \%$ para hemangiomas superficiales, y encontraron una eficacia similar para ambos. Sin embargo, el tratamiento con imiquimod se asoció con diversos efectos adversos locales, como irritación, cicatrices, pigmentación cutánea y formación de costras (el más frecuente), pero no se relacionó con efectos sistémicos. Por su parte, Martínez, et al. ${ }^{20}$ utilizaron imiquimod al $5 \%$ para el manejo de dos pacientes, de 7 y 14 meses de edad, con HI superficial. El tratamiento se aplicó tres veces por semana, alternando con 2 semanas libres de tratamiento para disminuir los efectos inflamatorios locales. Se logró regresión del HI en ambos pacientes y los efectos adversos fueron similares a los previamente reportados.

$\mathrm{Ho}$, et al. ${ }^{21}$ realizaron un estudio retrospectivo en Toronto, Canadá, con una muestra más amplia. Incluyeron 18 niños con una edad promedio de 18 semanas. Se trataron 22 hemangiomas con diferentes topografías (la mayoría localizados en la cabeza) con imiquimod al $5 \%$ crema, que se aplicó de tres a cinco veces por semana durante un promedio de 17 semanas (7 a 46 semanas). Se observó la remisión de cuatro hemangiomas y la reducción en el tamaño de todos los hemangiomas superficiales. Se trató un hemangioma ulcerado y se observó la reducción en el tamaño de la lesión y el aceleramiento en la reparación de la úlcera. Los hemangiomas profundos y mixtos no mostraron mejoría después del tratamiento. Los efectos secundarios fueron similares a los reportados: los más comunes fueron la irritación y la formación de costras. Aunque se trata de un estudio retrospectivo, los autores concluyeron que el imiquimod al $5 \%$ en crema 
puede ser efectivo en el manejo de hemangiomas superficiales en pacientes pediátricos.

En otro estudio más reciente, realizado en Canadá por McCuaig, et al. ${ }^{22}$, se valoró la eficacia de imiquimod para el tratamiento de $\mathrm{HI}$ superficiales y mixtos. Con una muestra de 16 pacientes y un promedio de edad de 4 meses, se aplicó imiquimod al 5\% en crema de tres a siete veces por semana por 16 semanas. A los 4 meses de seguimiento se reportó una mejoría en la coloración, pero ningún paciente presentó reducción en el tamaño del $\mathrm{HI}$, lo que sugiere que los efectos del imiquimod se limitan a los hemangiomas con componente superficial. No se reportó toxicidad sistémica.

A la fecha, no existe una dosis estándar de imiquimod para el tratamiento efectivo de hemangiomas debido a los escasos reportes en la literatura. El tratamiento con imiquimod puede ser una alternativa en el manejo de hemangiomas superficiales, aunque presenta más efectos adversos que otros fármacos.

\section{Corticosteroides tópicos}

En series pequeñas, se han utilizado corticosteroides tópicos clase I y clase II con buenos resultados. Estos fármacos se pueden utilizar para el tratamiento de hemangiomas planos o placas mínimamente elevadas en sitios con predisposición a ulceración o desfiguración $n^{14}$. Sin embargo, su eficacia es menor a la de los corticosteroides intralesionales. Dentro de los efectos adversos reportados se encuentran atrofia, telangiectasias, ulceración, estrías, hipopigmentación, hipertricosis e infecciones. Las formulaciones que se han utilizado incluyen propionato de clobetasol al $0.05 \%$, dipropionato de betametasona al $0.05 \%$, propionato de halobetasol al $0.05 \%$ y furoato de mometasona al $0.1 \%$, aplicados dos veces al día por periodos de 4 a 21 semanas. En general, son una opción relativamente segura para lesiones en cualquier sitio, incluyendo la región periocular, pero son menos efectivos que otras opciones tópicas como el timolol ${ }^{23,24}$.

\section{Brimonidina}

La brimonidina es un agonista adrenérgico $\alpha-2$, por lo que tiene un potente efecto vasoconstrictor. Fue aprobado por la Food and Drug Administration (FDA), en el 2007, para el tratamiento de glaucoma en mayores de 2 años de edad. Otras aplicaciones, no aprobadas por la FDA, incluyen su formulación en crema al $0.5 \%$ para el tratamiento de la rosáce ${ }^{25}$.
Este fármaco es útil para el tratamiento de $\mathrm{HI}$, ya que tiene un efecto en la vasculatura periférica anormal. Se ha utilizado en combinación con timolol tópico, en una fórmula de brimonidina al $0.2 \%$-timolol al $0.5 \%$, con lo que se ofrece un efecto sinérgico contra el crecimiento de los hemangiomas (el efecto $\beta$-bloqueador no selectivo que ofrece el timolol y el efecto $\alpha-2$ adrenérgico de la brimonidina). A la fecha, su aplicación en hemangiomas ha tenido buenos resultados en algunas series y reportes de casos, en los que ha reducido el tamaño y el componente papular y ha aclarado la coloración de la lesión. Se recomienda la aplicación de una a dos gotas cada $12 \mathrm{~h}$ con la punta del dedo. Se debe aplicar con precaución para evitar su absorción sistémica, sobre todo en hemangiomas perioculares. En este caso, se recomienda aplicar el tratamiento con los párpados cerrados, ocluyendo el canto medial hasta $60 \mathrm{~s}$ después de la aplicación ${ }^{26,27}$.

Los efectos secundarios más comúnmente reportados con el uso de brimonidina incluyen mareo, letargia, fatiga, hipotermia, bradicardia e hipotensión. Sin embargo, se reportó un caso de una paciente de 2 meses de edad, con un HI ulcerado que afectaba el párpado, quien presentó toxicidad a la brimonidina y desarrolló deterioro respiratorio (episodios de apnea) y neurológico (letargia e hipotonía) ${ }^{28}$.

La vida media de la administración oftálmica de este medicamento es de 2 a $3 \mathrm{~h}$. Sin embargo, se desconoce la farmacocinética cuando se usa de forma tópica. Se han reportado efectos adversos graves similares en otros estudios, sobre todo al usarse brimonidina en menores de 3 años de edad ${ }^{29}$. Aunque la brimonidina parece ser una terapia alternativa prometedora, son necesarios estudios adicionales para la evaluación de sus efectos adversos severos, especialmente en la población infantil.

\section{Láseres}

El láser de colorante pulsado produce fototermólisis selectiva, con lo cual los vasos sanguíneos son destruidos en forma selectiva, y causan daño mínimo al tejido cercano. Se trata de la modalidad de láser más utilizada en el tratamiento de $\mathrm{HI}$, que penetra solamente hasta $1.2 \mathrm{~mm}$ de profundidad en la piel, por lo que está indicado en el tratamiento de lesiones muy superficiales, ulceradas o residuales como eritema o telangiectasias ${ }^{30}$. Su uso en hemangiomas no complicados y en fases proliferativas es muy controversial. Se han realizado múltiples estudios comparativos donde no se ha encontrado ninguna diferencia clínica significativa entre el uso de 
Tabla 2. Principales tratamientos sistémicos de hemangiomas infantiles ${ }^{40,44,49-53}$

\begin{tabular}{|c|c|c|}
\hline Fármaco & Dosis & Efectos adversos \\
\hline Propranolol & $\begin{array}{l}3 \mathrm{mg} / \mathrm{kg} / \mathrm{día} \text { administrado dos a tres veces } \\
\text { al día } \\
\text { Se inicia con } 1 \mathrm{mg} / \mathrm{kg} / \text { día y se va } \\
\text { escalando la dosis }\end{array}$ & $\begin{array}{l}\text { Comunes: alteraciones del sueño, como pesadillas o terrores nocturnos, } \\
\text { y acrocianosis } \\
\text { Raros: hipoglucemia y broncoespasmo }\end{array}$ \\
\hline Nadolol & $2.19 \pm 1.1 \mathrm{mg} / \mathrm{kg} / \mathrm{día}$ & $\begin{array}{l}\text { Raras: Alteraciones del sueño, alteraciones gastrointestinales leves y } \\
\text { acrocianosis }\end{array}$ \\
\hline Atenolol & $1 \mathrm{mg} / \mathrm{kg} / \mathrm{día}$ & Diarrea, agitación, alteraciones del sueño y bradicardia \\
\hline Prednisona & $2-3 \mathrm{mg} / \mathrm{kg} / \mathrm{día}$ & $\begin{array}{l}\text { Supresión del eje hipotálamo-hipófisis, síndrome de Cushing, retraso del } \\
\text { crecimiento, aumento de peso, hipertensión arterial, enfermedad } \\
\text { ácido-péptica, irritabilidad, insomnio, inmunosupresión, cardiomiopatía, } \\
\text { osteopenia, glaucoma y cataratas }\end{array}$ \\
\hline
\end{tabular}

este tipo de láser y la modalidad de vigilancia en hemangiomas no complicados, más allá de mejores resultados cosméticos. Sin embargo, se ha relacionado con la presencia de más efectos adversos como hipopigmentación, cicatrices y cambios en la textura de la piel. En la actualidad, los criterios del uso de láser en HI no están bien definidos, así que ha quedado restringido al tratamiento de lesiones ulceradas que no responden a tratamiento médico o de lesiones residuales ${ }^{31}$.

Por otro lado, el láser Nd: YAG, que penetra a mayor profundidad en la piel, resultó ser menos efectivo y con más efectos adversos que el láser de colorante pulsado en un estudio retrospectivo realizado por Raulin, et al. ${ }^{32}$, que incluyó 50 pacientes con hemangiomas superficiales. Sin embargo, esta modalidad de láser se puede utilizar después del tratamiento inicial con láser de colorante pulsado, para optimizar los resultados cosméticos.

Se ha reportado el uso de la terapia con láser intralesional KTP (Potasio-Titanil-Fosfato) con excelentes resultados, en especial en el manejo de hemangiomas voluminosos: se coloca una fibra óptica en el interior de la lesión y se administra el láser. A pesar de sus excelentes resultados, los riesgos de efectos adversos son mayores: lesión a nervios periféricos, necrosis cutánea y ulceraciones, que se han reportado hasta en el $20 \%$ de los casos $^{33}$.

\section{Tratamiento sistémico}

A inicios de 1960, los corticosteroides sistémicos e intralesionales eran considerados la piedra angular en el tratamiento de los HI. La reducción del tamaño de los $\mathrm{HI}$ con corticosteroides sistémicos se descubrió por casualidad en los pacientes con hemangioendoteliomas tratados por trombocitopenia en las décadas de los 50 y los $60^{34}$. Posteriormente, en las décadas de los 80 y 90 , el IFN- $\alpha$ mostró efectividad en algunos $\mathrm{HI}$ resistentes al tratamiento con esteroides sistémicos ${ }^{35}$. Sin embargo, hoy en día, se conoce su alta toxicidad neurológica, por lo que no se utiliza como tratamiento de primera línea.

En 2008, Léauté-Labrèze, et al. ${ }^{36}$ reportaron -por serendipia- que el propranolol, un bloqueador no selectivo de receptores $\beta$-adrenérgicos utilizado por décadas para trastornos cardíacos en niños, era efectivo y bien tolerado en el tratamiento de los HI. Un año después, reportaron su experiencia en 32 niños con $\mathrm{HI}$ grandes que fueron tratados con propranolol a una dosis de $2-3 \mathrm{mg} / \mathrm{kg} / \mathrm{día}$, dividido en dos a tres dosis ${ }^{37}$.

\section{Bloqueadores $\beta$-adrenérgicos}

Se considera el tratamiento de primera línea en $\mathrm{HI}$ complicados (Tabla 2). A pesar de las preocupaciones iniciales de su seguridad clínica, estudios recientes sugieren que, para la mayoría de los pacientes, el propranolol es seguro, bien tolerado y efectivo ${ }^{38,39}$.En EE.UU. existe una presentación oral en solución desarrollada para su uso en niños, que fue aprobada por la FDA en 2014 con el nombre de Hemangeol ${ }^{40}$.

El mecanismo de acción del propranolol en los $\mathrm{HI}$ aún es desconocido. Existen varias teorías y mecanismos de acción propuestos: vasoconstricción, inhibición de la angiogénesis vía supresión del factor de crecimiento endotelial vascular alfa (VEGF- $\alpha$ ) y disminución de metaloproteinasas e IL-6, regulación del eje renina-angiotensina e inhibición de la producción del óxido nítrico ${ }^{41-43}$. Se han encontrado receptores $\beta$-adrenérgicos en las células endoteliales de capilares de $\mathrm{HI}$ 
proliferativos y VEGF elevados en $\mathrm{HI}$ de rápido crecimiento que son suprimidos por el propranolol ${ }^{6}$.

Existen múltiples ensayos clínicos controlados acerca del uso de propranolol. Uno de los más grandes incluyó 460 niños entre 1 y 5 meses de edad, a quienes se les administró una dosis de $3.4 \mathrm{mg} / \mathrm{kg} /$ día del fármaco. Los niños mostraron una tasa de mejoría del $60 \%$ con una resolución completa o casi completa del hemangioma, comparado con una tasa de éxito del $4 \%$ en aquellos tratados con placebo ${ }^{44}$. En general, se ha encontrado una respuesta positiva en el $85 \%$ de las publicaciones y una tasa de falla de respuesta al tratamiento del $1.6 \%{ }^{46}$. En un metaanálisis reportado en 2013, se incluyeron 35 estudios con $324 \mathrm{HI}$, donde la eficacia del propranolol fue mayor que la del resto de los tratamientos, incluyendo esteroides sistémicos, vincristina y láser, en hemangiomas cutáneos, perioculares, en vía aérea y hepáticos ${ }^{46}$.

El aclaramiento del color y mejoría en la textura se observa después de pocas horas o días del inicio del tratamiento en la mayoría de los casos. Al igual que los esteroides sistémicos, el propranolol estabiliza la fase de crecimiento de los HI; sin embargo, también es efectivo después de la fase proliferativa (Fig. 2) ${ }^{47}$.

Todo paciente que va a iniciar tratamiento con propranolol necesita una valoración cardiovascular, así como un electrocardiograma (ECG). En algunos casos, puede realizarse por el mismo pediatra, aunque algunos médicos prefieren el diagnóstico de un cardiólogo antes de iniciar el tratamiento con el fármaco. Sin embargo, esta indicación parece ser de valor limitado en pacientes sin historia personal de alteraciones cardia$\mathrm{cas}^{48}$. Algunos autores recomiendan la realización del ECG previo al inicio del tratamiento en las siguientes situaciones ${ }^{49}$ :

- Cuando la frecuencia cardiaca se encuentra por debajo de lo normal para la edad del paciente: en recién nacidos, < 70 latidos por minuto; lactantes (1-12 meses), $<80$ latidos por minuto; niños (> 12 meses de edad), $<70$ latidos por minuto.

- Historia familiar de enfermedades cardiacas congénitas o arritmias (bloqueos, síndrome de QT largo, muerte súbita) o historia de enfermedades de tejido conectivo en la madre.

- Antecedente de arritmia o arritmia durante la exploración física.

La dosis inicial recomendada de propanolol es de $1 \mathrm{mg} / \mathrm{kg} / \mathrm{día}$. Esta dosis se va escalando hasta llegar a $3 \mathrm{mg} / \mathrm{kg} / \mathrm{día}$; la dosis máxima es de $3.4 \mathrm{mg} / \mathrm{kg} /$ día. Se recomienda su administración de 2 a 3 veces al día. Al iniciar el tratamiento, se debe medir la presión

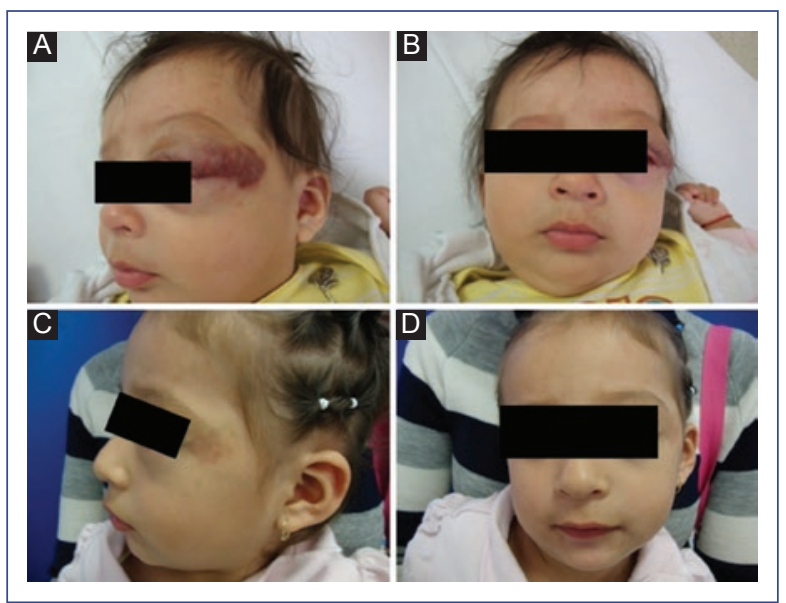

Figura 2. Hemangioma infantil mixto en párpado superior izquierdo. A y B: previo al tratamiento con propranolol. C y D: después de 1 año y 9 meses de tratamiento con propranolol.

arterial basal 1 a $2 \mathrm{~h}$ después de la primera dosis, y 1 a $2 \mathrm{~h}$ después de cada incremento de $0.5 \mathrm{mg} / \mathrm{kg}$ en la dosis. Si la presión arterial o la frecuencia cardiaca disminuyen más de 2 desviaciones estándar del promedio, se sugiere interconsulta con cardiología ${ }^{44}$. Se recomienda que los pacientes $<8$ semanas de vida, pretérmino $<48$ semanas posconcepción, aquellos con bajo nivel socioeconómico o con factores de riesgo cardiovascular o pulmonar sean hospitalizados para iniciar el tratamiento ${ }^{49}$.

Las contraindicaciones para el uso del propranolol incluyen choque cardiogénico, bradicardia sinusal, hipotensión, bloqueo cardiaco grado $2 \circ 3$, insuficiencia cardiaca, asma o hipersensibilidad al fármaco. Se debe tener especial cuidado en aquellos pacientes con síndrome de PHACES (acrónimo correspondiente a malformaciones de la fosa Posterior, Hemangioma facial gigante, defectos Arteriales, Cardiacos y coartación de la aorta, alteraciones oculares (Eye) y del desarrollo ventral que incluye hendidura eSternal o del rafe supraumbilical) por riesgo de eventos vasculares cerebrales ${ }^{49}$.

Los principales efectos secundarios del propanolol son alteraciones del sueño, como pesadillas o terrores nocturnos, y acrocianosis. En algunos casos se puede presentar hipotensión o bradicardia, que casi siempre son asintomáticas; otras complicaciones raras son la hipoglucemia y el broncoespasmo. La hipoglucemia sintomática y las convulsiones secundarias a hipoglucemia se han observado en pacientes con baja ingesta de alimentos o con una infección concomitante. El riesgo de hipoglucemia disminuye al alimentar de 
manera regular al paciente y evitar ayunos prolongados. Los pacientes con enfermedad de vías respiratorias con riesgo de broncoespasmo también deben suspender el tratamiento por unos días ${ }^{49,50}$.

La mejoría más importante con el uso de propranolol ocurre en los primeros 3 a 4 meses después del inicio del tratamiento. Usualmente, continúa por lo menos hasta los 8 a 12 meses de edad, o 3 a 12 meses de terapia. Para suspenderlo, se debe disminuir gradualmente la dosis durante un periodo de 1 a 3 semanas, para prevenir taquicardia de rebote ${ }^{45}$.

\section{Nadolol}

El nadolol es un $\beta$-bloqueador sintético no selectivo que se considera más seguro que el propranolol debido a que no atraviesa la barrera hematoencefálica (BHE). La vida media del nadolol es más larga que la del propranolol, por lo que su administración es solamente una vez al día. Pope, et al. ${ }^{40}$ realizaron un estudio comparativo del uso de propranolol con el de nadolol en $\mathrm{HI}$, donde incluyeron diez pacientes en tratamiento con nadolol (dosis media de $2.19 \pm 1.1 \mathrm{mg} / \mathrm{kg} /$ día) y nueve con propranolol $(1.89 \pm 0.29 \mathrm{mg} / \mathrm{kg} / \mathrm{día})$. A las 24 semanas de tratamiento, se evidenció una mayor disminución del tamaño del hemangioma en el grupo de nadolol $(97 \pm$ $3.05 \%$ ) en comparación con el grupo de propranolol (86 $\pm 14.82 \% ; p<0.001$ ). El nadolol fue bien tolerado en general, aunque se presentaron alteraciones del sueño (un paciente), alteraciones gastrointestinales leves (cinco pacientes) y extremidades frías (dos pacientes). Se considera que la vida media más larga del nadolol disminuye el riesgo de rebote $\mathrm{y}$, como no atraviesa la $\mathrm{BHE}$, no causa terrores nocturnos ni preocupación sobre la pérdida de memoria. El nadolol se considera un tratamiento seguro y efectivo para el tratamiento del $\mathrm{HI}$. Sin embargo, no se cuenta con este fármaco en México.

\section{Atenolol}

Es un bloqueador- $\beta 1$ sistémico hidrofílico, más selectivo que el propranolol, lo que le confiere un mejor perfil de seguridad. Se ha reportado su uso exitoso en niños que no toleraron la administración de propranolol. Sin embargo, su tiempo de respuesta es más lento comparado con el propranolol, y no es tan eficaz en disminuir la coloración de las lesiones. La dosis sugerida es de $1 \mathrm{mg} / \mathrm{kg} / \mathrm{dí}$. Sus principales efectos adversos son diarrea, agitación, alteraciones del sueño y bradicardia; esta última se presenta después de $1 \mathrm{a}$ $2 \mathrm{~h}$ de la ingesta del medicamento y es revertida espontáneamente a la normalidad tras este tiempo. No se han reportado eventos adversos graves como hipoglicemia, broncoespasmo o hipotensión. A pesar de que el efecto de bloqueador- $\beta 2$ a dosis pequeñas es poco probable, se recomienda que se realicen los mismos estudios para descartar las alteraciones cardíacas observadas con el uso de propranolol ${ }^{51-53}$.

$\mathrm{Ji}$, et al. ${ }^{54}$ realizaron un estudio en el Hospital West China de la Universidad Sichuan, en 2016, que incluyó 70 pacientes con diagnóstico de $\mathrm{HI}$ de 5 a 20 semanas de edad. Todos recibieron evaluación cardiovascular previa y, posteriormente, fueron tratados con atenolol a una dosis de $1 \mathrm{mg} / \mathrm{kg} / \mathrm{día}$ durante 24 semanas. Se observó la detención del crecimiento del hemangioma de manera abrupta a las 4 semanas en el $93.4 \%$ de los pacientes; a los 3 meses, se observó la regresión, al menos, del $80 \%$ de la lesión. Los efectos adversos más comunes fueron diarrea, agitación, bradicardia no sostenida, bronquiolitis y alteraciones en el sueño. Una de las pacientes tuvo que suspender el tratamiento debido a enteritis hemorrágica, aunque no se aclaró si este evento fue una manifestación gastrointestinal de la administración de atenolol o un evento incidental. Como conclusión, este estudio mostró que el atenolol oral tiene un efecto positivo, ya que reduce la progresión de los $\mathrm{HI}$ y ofrece un perfil seguro para la población infantil.

\section{Corticosteroides sistémicos}

El tratamiento con esteroides sistémicos ha sido sustituido por el propranolol. Sin embargo, estos fármacos tuvieron un papel muy importante en el pasado y continúan siendo una opción de tratamiento para aquellos pacientes que no pueden tomar $\beta$-bloqueadores. Una revisión sistemática de la literatura reportó una tasa de respuesta del $84 \%$ a una dosis diaria de prednisona de $2.9 \mathrm{mg} / \mathrm{kg}^{55}$.

Por otra parte, el tratamiento con esteroides muestra también una alta tasa de recaída, de hasta el 37\%. La dosis recomendada es de 2-3 mg/kg/día y la duración depende de la tasa de respuesta: usualmente es de 4 a 12 semanas a dosis normales y, posteriormente, se inicia un esquema de reducción muy lento de hasta 12 meses $^{5}$. Los efectos secundarios de los esteroides sistémicos son ampliamente conocidos, entre los que destacan la supresión del eje hipotálamo-hipófisis, síndrome de Cushing, retraso del crecimiento, aumento de peso, hipertensión arterial, enfermedad ácido-péptica, irritabilidad, insomnio, inmunosupresión, cardiomiopatía, osteopenia, glaucoma y cataratas ${ }^{56}$. Se realizó un ensayo clínico controlado de no inferioridad en el 
Hospital Universitario de Seúl, en el que compararon la eficacia y seguridad entre el propranolol y los esteroides sistémicos. Se incluyeron a 34 pacientes con una edad promedio de 3.3 meses y fueron aleatoriamente asignados al grupo de propranolol o de esteroide sistémico. A 16 semanas de tratamiento, la tasa de respuesta del grupo de propranolol fue del $95.7 \%$ y del grupo de esteroides, del 91.9\%. Este estudio demostró que el efecto terapéutico del propranolol no es inferior al de los esteroides sistémicos ${ }^{57}$.

\section{Otros tratamientos sistémicos}

La vincristina es un alcaloide que impide la mitosis vía la formación de microtúbulos e induce apoptosis de células endoteliales. Se ha utilizado para hemangiomas grandes que ponen en peligro la vida (vía aérea, orbitarios o hepáticos). Sin embargo, presenta muchos efectos secundarios y no se considera tratamiento de primera línea ${ }^{58}$.

Otro tratamiento utilizado es el IFN- $\alpha 2 a \circ 2 b$. Se administra de manera subcutánea y se debe mantener bajo vigilancia con biometría hemática y pruebas de función hepática. El tratamiento dura de 2 a 12 meses. Se ha reportado una tasa de respuesta del $90 \%$ en pacientes resistentes a esteroides sistémicos; sin embargo, se ha observado una alta tasa de rebote y efectos secundarios, como la diplegia espástica, por lo que se considera como último recurso de tratamiento ${ }^{6}$.

\section{Tratamiento quirúrgico}

En caso de ser necesario, el tratamiento quirúrgico se recomienda al terminar la fase proliferativa del hemangioma, cuando este haya involucionado el máximo posible y solamente quede tejido residual fibroadiposo. Sin embargo, en pacientes con contraindicación para el tratamiento farmacológico, el tratamiento quirúrgico puede ser una buena opción terapéutica ${ }^{59}$.

Como conclusiones, se puede decir que la decisión del tratamiento y la mejor opción terapéutica para un paciente con $\mathrm{HI}$ debe individualizarse dependiendo de varios factores, como el tamaño de la lesión, la localización, la presencia de complicaciones como ulceración, el riesgo de cicatrización o desfiguramiento, la edad del paciente, la tasa de crecimiento o de involución al momento del diagnóstico, los riesgos y beneficios de administrar el tratamiento, la disponibilidad del medicamento, los costos y la experiencia del médico tratante.

El tratamiento está indicado en $\mathrm{HI}$ complicados que interfieran con alguna estructura o función vital y para lesiones de gran tamaño con riesgo de ulceración, cicatrización o desfiguramiento. Las metas del tratamiento son el prevenir complicaciones, riesgo de sangrado, infección, dolor, mejoría cosmética y minimizar el estrés psicosocial que vive la familia y el paciente. El propranolol es, en la actualidad, el tratamiento de primera línea para $\mathrm{HI}$ complicados. En caso de hemangiomas no complicados, la decisión de iniciar una intervención terapéutica será del médico tratante y de la familia del paciente.

\section{Responsabilidades éticas}

Protección de personas y animales. Los autores declaran que para esta investigación no se han realizado experimentos en seres humanos ni en animales.

Confidencialidad de los datos. Los autores declaran que han seguido los protocolos de su centro de trabajo sobre la publicación de datos de pacientes.

Derecho a la privacidad y consentimiento informado. Los autores han obtenido el consentimiento informado de los pacientes y sujetos referidos en el artículo. Este documento obra en poder del autor de correspondencia.

\section{Agradecimientos}

Al servicio de Dermatología del Instituto Nacional de Pediatría, Ciudad de México, México.

\section{Conflicto de intereses}

Los autores declaran no tener ningún conflicto de intereses.

\section{Financiamiento}

Ninguno.

\section{Bibliografía}

1. Kilcline C, Frieden IJ. Infantile hemangiomas: how common are they? A systematic review of the medical literature. Pediatr Dermatol. 2008;25: 168-73.

2. Hoornweg MJ, Smeulders MJ, Ubbink DT, Van Der Horst CM. The prevalence and risk factors for infantile haemangiomas: a case-control study in the Dutch population. Paediatr Perinat Epidemiol. 2012;26:156-62.

3. Haggstrom AN, Drolet BA, Baselga E, Chamlin SL, Garzon MC, Horii KA, Hemangioma Investigator Group. Prospective study of infantile hemangiomas: demographic, prenatal and perinatal characteristics. J Pediatr. 2007;150:291-4.

4. Giachetti A, Sojo MM, García-Mónaco R. Hemangiomas infantiles. Arch Argent Pediatr. 2013;111:537-45.

5. Drolet BA, Swanson EA, Frieden IJ, Hemangioma Investigator Group. Infantile hemangiomas: an emerging health issue linked to an increased rate of low birth weight infants. J Pediatr. 2008;153:712-5.

6. Darrow DH, Greene AK, Mancini AJ, Nopper AJ, Section on dermatology, section on otolaryngology — head and neck surgery, and section on plastic surgery. Diagnosis and management of infantile hemangioma. Pediatrics. 2015;136:e1060-104. 
7. Chang LC, Haggstrom AN, Drolet BA, Baselga E, Chamlin SL, Garzon MC Hemangioma Investigator Group. Growth characteristics of infantile hemangiomas: implications for management. Pediatrics. 2008;122:360-7.

8. Smithson SL, Rademaker M, Adams S, Bade S, Bekhor P, Davidson S, et al. Consensus statement for the treatment of infantile haemangiomas with propranolol. Australas J Dermatol. 2017;58:155-9.

9. Guo S, Ni N. Topical treatment for capillary hemangioma of the eyelid using beta-blocker solution. Arch Ophthalmol. 2010;128:255-6.

10. Ni N, Langer P, Wagner R, Guo S. Topical timolol for periocular hemangioma: Report of further study. Arch Ophthalmol. 2011;129:377-9.

11. Semkova K, Kazandjieva J. Topical timolol maleate for treatment of infantile haemangiomas: Preliminary results of a prospective study. Clin Exp Dermatol. 2013;38:143-6.

12. Fernández-Ballesteros MD, Vera-Casaño A, Escudero-Gómez J, Hiraldo-Gamero A. Infantile hemangioma of the eyelid treated with timolol gel. Actas Dermosifiliogr. 2012;103:444-6.

13. Chan H, McKay C, Adams S, Wargon O. RCT of timolol maleate gel for superficial hemangiomas in 5-to 24 weeks-olds. Pediatrics. 2013;131:1739-47.

14. Sethuraman G, Yenamandra V, Gupta V. Management of infantile hemangiomas: Current Trends. J Cutan Aesthet Surg. 2014:7:75-85.

15. Kunzi-Rapp K. Topical propranolol therapy for infantile hemangiomas. Pediatr Dermatol 2012;29:154-9.

16. Xu G, Lv R, Zhao Z, Huo R. Topical propranolol for treatment of superficial infantile hemangiomas. J Am Acad of Dermatol. 2012:67:1210-3.

17. Kovačević M, Lukinović V, Maričić G, Krnjević-Pezić G, Stanimirović A. Topical propranolol cream in treatment of superficial infantile hemangiomas: a literature review and 4 years of clinical experience. Acta Dermatovenerol Alp Pannonica Adriat. 2014;23:75-8.

18. Li WV, Li W, Talcott EK, Zhai W. Imiquimod as an antiangiogenic agent. J Drugs Dermatol. 2005;4:708-17.

19. Qiu $\mathrm{Y}, \mathrm{Ma} \mathrm{G}$, Yang J, $\mathrm{Hu} \mathrm{X}$, Chen $\mathrm{H}$, Jin $\mathrm{Y}$, et al. Imiquimod $5 \%$ vs cream versus timolol $0.5 \%$ ophthalmic solution for treating superficial proliferating infantile haemangiomas: a retrospective study. Clin Exp Dermatol. 2013;38:845-50

20. Martínez MI, Sánchez-Carpintero I, North PE, Mihm MC Jr. Infantile hemangioma: Clinical resolution with $5 \%$ imiquimod cream. Arch dermatol. 2002;138:881-4.

21. Ho NT, Lansang P, Pope E. Topical imiquimod in the treatment of infantile hemangiomas: a retrospective study. J Am Acad Dermatol. 2007;56:63-8.

22. McCuaig CC, Dubois J, Powell J, Belleville C, David M, Rousseau E, et al. A phase II, open-label study of the efficacy and safety of imiquimod in the treatment of superficial and mixed infantile hemangioma. Pediatr Dermatol. 2009;26:203-12.

23. Elsas FJ, Lewis AR. Topical treatment of perioocular capillary hemangioma. J Pediatr Ophtalmol Strabismus. 1994;31:4-7.

24. Garzon MC, Lucky AW, Hawrot A, Frieden IJ. Ultrapotent topical corticosteroid treatment of hemangiomas of infancy. J Am Acad Dermatol. 2005:52:281-6.

25. Fowler J, Jarrat M, Moore A, Meadows K, Pollack A, Steinhoff M, et al. Once daily topical brimonidine tartrate gel $0.5 \%$ is a novel treatment for moderate to severe facial erythema of rosacea: results of two multicentre, randomized and vehicle controlled studies. Br J Dermatol. 2012;166:633-41.

26. Chu MB, Searcy G, Siegfried E. Efficacy of topical brimonidine-timolol for haemangioma of infancy and perils of off-label prescribing. BMJ Case Rep. 2013: bcr2013009365. doi: 10.1136/bcr-2013-009365.

27. Beal BT, Chu MB, Siegfried EC. Ulcerated infantile hemangioma: nove treatment with topical brimonidine-timolol. Pediatr Dermatol 2014:31:754-6.

28. Gil K, Bayart C, Desai R, Golden A, Raimer P, Tamburro J. Brimonidine toxicity secondary to topical use for an ulcerated hemangioma. Pediatr Dermatol. 2016:33:232-4.

29. Lai Becker M, Huntington N, Woolf AD. Brimonidine tartrate poisoning in children: frequency, trends, and use of naloxone as an antidote. Pediatrics. 2009;123:e305-11.

30. Sánchez-Carpintero I, Mihm MC, Waner M. [Laser and intense pulsed light in the treatment of infantile haemangiomas and vascular malformations]. An Sist Sanit Navar. 2004;27:103-15.

31. Poetke M, Philipp C, Berlien HP. Flashlamp-pumped pulsed dye laser for hemangiomas in infancy: treatment of superficial vs. mixed hemangiomas. Arch Dermatol. 2000;136:628-32.

32. Raulin C, Greve B. Retrospective clinical comparison of hemangioma treatment by flashlamp-pumped $(585 \mathrm{~nm})$ and frequency-doubled Nd: YAG (532 nm) lasers. Lasers Surg Med. 2001;28:40-3.

33. Burstein FD, Williams JK, Schwentker AR, Nahai F. Intralesional laser therapy treatment for hemangiomas: technical evolution. J Craniofac Surg. 2006;17:756-60
34. Katz HP, Askin J. Multiple hemangiomata with thrombopenia: an unusual case with comments on steroid therapy. Am J Dis Child. 1968;115:351-7.

35. Ezekowitz RA, Mulliken JB, Folkman J. Interferon alfa-2a therapy for life-threatening hemangiomas of infancy. N Engl J Med. 1992;326: 1456-63.

36. Léauté-Labrèze $\mathrm{C}$, Dumas de la Roque $\mathrm{E}$, Hubiche T, Boralevi F, Thambo JB, Taïeb A. Propranolol for severe hemangiomas of infancy. N Engl Med. 2008;358:2649-51.

37. Sans V, de la Roque ED, Berge J, Grenier N, Boralevi F, Mazereeuw-Hautier J, et al. Propranolol for severe infantile hemangiomas: follow-up report. Pediatrics 2009;124:423-31.

38. Liu LS, Sokoloff D, Antaya RJ. Twenty-four-hour hospitalization for patients initiating systemic propranolol therapy for infantile hemangiomasis it indicated? Pediatr Dermatol. 2013:30:554-60.

39. Hogeling M, Adams S, Wargon O. A randomized controlled trial of propranolol for infantile hemangiomas. Pediatrics. 2011;128:e259-66.

40. Pope E, Chakkittakandiyil A, Lara-Corrales I, Maki E, Weinstein M. Expanding the therapeutic repertoire of infantile haemangiomas: cohort-blinded study of oral nadolol compared with propranolol. $\mathrm{Br} J$ Dermatol. 2013;168:222-4.

41. Itinteang T, Withers AH, Davis PF, Tan ST. Biology of infantile hemangioma. Front Surg. 2014;1:38.

42. Dai Y, Hou F, Buckmiller L, Fan CY, Saad A, Suen J, et al. Decreased eNOS protein expression in involuting and propranolol-treated hemangiomas. Arch Otolaryngol Head Neck Surg. 2012;138:177-82.

43. Ji Y, Chen S, Xu C, Li L, Xiang B. The use of propranolol in the treatment of infantile haemangiomas: an update on potential mechanisms of action. Br J Dermatol. 2015:172:24-32.

44. Léauté-Labrèze C, Hoeger P, Mazereeuw- Hautier J, Guibaud L, Baselga E, Posiunas G, et al. A randomized, controlled trial of oral propranolol in infantile hemangioma. N Engl J Med. 2015;372:735-46.

45. Starkey E, Shahidullah H. Propranolol for infantile haemangiomas: a review. Arch Dis Child. 2011;96:890-3.

46. Lou Y, Peng WJ, Cao Y, Cao DS, Xie J, Li HH. The effectiveness of propranolol in treating infantile haemangiomas: a meta-analysis including 35 studies. Br J Clin Pharmacol. 2014:78:44-57.

47. Vivas-Colmenares GV, Bernabeu-Wittel J, Alonso-Arroyo V, Matute de Cardenas JA, Fernandez-Pineda I. Effectiveness of propranolol in the treatment of infantile hemangioma beyond the proliferation phase. Pediatr Dermatol. 2015;32:348-52

48. Raphael MF, Breugem CC, Vlasveld FA, de Graaf M, Slieker MG, Pasmans SG, et al. Is cardiovascular evaluation necessary prior to and during beta-blocker therapy for infantile hemangiomas? A cohort study. J Am Acad Dermatol. 2015;72:465-72.

49. Drolet BA, Frommelt PC, Chamlin SL, Haggstrom A, Bauman NM, Chiu YE, et al. Initiation and use of propranolol for infantile hemangioma: report of a consensus conference. Pediatrics. 2013;131:128-40.

50. Blei F, McElhinney DB, Guarini A, Presti S. Cardiac screening in infants with infantile hemangiomas before propranolol treatment. Pediatr Dermatol. 2014;31:465-70.

51. Raphael MF, de Graaf M, Breugem CC, Pasmans SGMA, Breur JMPJ, et al. Atenolol: a promising alternative to propranolol for the treatment of hemangiomas. J Am Acad Dermatol. 2011:65:420-1.

52. De Graaf M, Raphael MF, Breugem CC, Knol MJ, Bruijnzeel-Koomen CA, Kon M, et al. Treatment of infantile haemangiomas with atenolol: comparison with a historical propranolol group. J Plast Reconstr Aesthet Surg. 2013;66:1732-40.

53. Abarzua-Araya A, Navarrete-Dechent CP, Heusser F, Retamal J, Zegpi-Trueba MS. Atenolol versus propranolol for the treatment of infantile hemangiomas: a randomized controlled study. J Am Acad Dermatol. 2014;70:1045-9.

54. Ji Y, Wang Q, Chen S, Xiang B, Xu Z, Li Y, et al. Oral atenolol therapy for proliferating infantile hemangioma: a prospective study. Medicine (Baltimore). 2016;95:e3908.

55. Bennett ML, Fleischer AB Jr, Chamlin SL, Frieden IJ. Oral corticosteroid use is effective for cutaneous hemangiomas: an evidence-based evaluation. Arch Dermatol. 2001:137:1208-13.

56. Kelly ME, Juern AM, Grossman WJ, Schauer DW, Drolet BA. Immunosuppressive effects in infants treated with corticosteroids for infantile hemangiomas. Arch Dermatol. 2010;146:767-74.

57. Kim KH, Choi TH, Choi Y, Park YW, Hong KY, Kim DY, et al. Comparison of efficacy and safety between propranolol and steroid for infantile hemangioma: a randomized clinical trial. JAMA Dermatol. 2017:153:529-36.

58. Enjolras $\mathrm{O}$, Brevière GM, Roger G, Tovi M, Pellegrino B, Varotti E, et al. [Vincristine treatment for function- and life-threatening infantile hemangioma]. Arch Pediatr. 2004;11:99-107.

59. Greene AK. Management of hemangiomas and other vascular tumors. Clin Plast Surg. 2011;38:45-63. 